\title{
How the use of segmentation signs in compound reading affects reading behavior: an eye-tracking study
}

\author{
Silvana Deilen \\ University of Mainz, \\ Germany
}

\author{
Silvia Hansen-Schirra \\ University of Mainz, \\ Germany
}

\author{
Arne Nagels \\ University of Mainz, \\ Germany
}

\begin{abstract}
Two eye-tracking experiments were conducted to investigate the effects of visual segmentation, complexity, and context on the cognitive processing of compounds in German Easy Language. By presenting compounds in different boundary conditions, we determined whether a segmentation cue facilitates the processing of compounds presented with and without contextual information. The study was conducted with unimpaired adults and with hearing-impaired pupils, representing one of the target groups of Easy Language. The results indicate that visual segmentation facilitates processing of compounds for pupils with low literacy skills. However, they only benefit from segmentation when morpheme boundaries are marked in a subtle way, i.e., without strikingly deviating from the standard version. Pupils with higher literacy skills and unimpaired adults do not profit from segmentation. Even though hyphenation slows down compound processing for unimpaired readers, initial processing advantages of hyphenated over concatenated compounds emerged, which is explained by the fact that hyphenation forces a morpheme-based access and enables fast recognition of the compound's first constituent. However, it hinders readers from accessing the compound via the direct route and thus slows down the processing of the compound as a whole. Furthermore, unimpaired readers and hearingimpaired pupils process compounds faster when presented with context.
\end{abstract}

Keywords: Eye tracking, Eye movement, Reading, Compound processing, Written language processing, Segmentation cues, German Easy Language, Reading skills, Individual differences, Hearing impairments

\section{Introduction}

In German, compounding is the most productive word formation process. Especially in noun-compounding, virtually any free noun can combine with any other to form a novel compound. German compounding is recursive, which means that the compound formation itself can again serve as the base for the word formation process, resulting in an even more complex structure (cf. Berg 2006). This "almost unconstrained ability for morphemes to be recombined to create new meaningful structures" (Libben 2008: 71) yields the existence of almost infinite compound words: A chair in the dining room is referred to as a diningroomchair [Esszimmerstuhl], and a show- room, in which these chairs are displayed, could be referred to as diningroomchairshowroom [Esszimmerstuhlausstellungsraum].

One of the main characteristics of German compounding is that even in these polymorphemic compounds (i.e., compounds consisting of at least three free morphemes) all constituents are generally written without segmentation cues like interword spaces or hyphens. This absence of segmentation cues makes it more difficult for the reader to process compounds, since the reader is unable to parafoveally parse compounds into their constituents (cf. Inhoff et al. 2000). As there is overwhelming evidence that the lexical processing system "seeks to maximize 
opportunity by extracting all possible morpheme sets from an input string and by constructing all possible morphological representations" (Libben 2006: 21), the absence of segmentation cues becomes even more problematic for processing compounds that by chance contain a morpheme which is not a constituent of the compound. These missing indications of the compound's branching structure are also problematic in trimorphemic compounds that theoretically allow more than one hierarchical option, specifically left-branching and rightbranching. In these compounds, the reader firstly has to figure out whether the middle part of the compound is associated with the first or the last constituent i.e., to construct its meaning, the reader has to assign a particular structure to the compound. These potential processing problems arising from concatenation of polymorphemic compounds are typical for German compounds. In English, for example, processing difficulties are minimized by an orthographic rule determining that, in trimorphemic compounds, an interword space must be present at the major morpheme boundary (cf. Libben 2008: 77). Consider for example the compound "Fußballschuhe" [football shoes]. While in German the reader does not immediately know whether "Fußballschuhe" is a left-branching or a right-branching compounds (i.e. whether the major morpheme boundary is between the second and the third [Fußball-Schuhe] or between the first and the second constituent [Fuß-Ballschuhe]), in English, the space at the major morpheme boundary (football shoes) indicates that the compound is left-branching, consequently telling the reader that "shoes" is modified by the first two constituents. Furthermore, in English, not only compounds with more than two constituents but also less familiar or novel bimorphemic compounds are usually written with interword spaces at the morpheme boundaries (cf. Inhoff et al. 2000). German, however, does not make use of orthographic conventions to convey the compound's structure.

Even though there is strong eye-tracking evidence that the processing of compounds requires the reader to identify and comprehend all single constituents (cf. Bertram et al. 2011; Placke 2001, Bertram/Hyönä 2003; Kuperman et al. 2009), there is little doubt that proficient readers do not have difficulties decoding complex concatenated compounds. When reading compounds with an ambiguous branching structure such as "Stadtparkplatz" (Stadt Parkplatz vs. Stadtpark Platz [a parking lot in the city vs. a place in the city park]), proficient readers make use of contextual information to identify the compound's correct major and minor morpheme boundaries and to assign the compound its intended meaning. The context, in which the compound is embedded, therefore disambiguates its meaning and helps guide the reader's choice of the appropriate meaning. However, the task of locating the correct morpheme boundary and doing so as quickly as possible may create substantial problems for people with reading impairments. This assumption was confirmed by several studies which provided evidence that people with reading impairments have more difficulties accessing compounds than monomorphemic words of equal length and frequency (Delazer/Semenza 1998; Blanken 2000; Lorenz 2008; Lorenz et al. 2014; Seyboth 2014). The main reason for the increased cognitive processing costs impaired readers face when reading compounds is that they have to parse compounds into their constituents, before being able to infer the meaning of the compound. The finding that people with reading impairments have a great deal of difficulty when segmenting compounds is also due to the fact that, as mentioned above, a large amount of German compounds happen to contain (pseudo-)morphemes in the orthography which are not constituents of the compound (cf. Libben 2006). To illustrate, the compound "Altbaucharme" [charm of old buildings] is made of the constituents "Alt", "Bau", and "Charme". However, the compound also contains two other free potential morphemes, namely "Bauch" and "Arme"; yet, neither of these is a constituent of the compound. Therefore, when reading compounds with ambiguous segmentation options, readers might encounter words that are known to them, which, however, do not form part of the compound. These pseudo-constituents are consequently a barrier to the identification of the constituents and the comprehension of the compound. In Easy Language, these "structural difficulties" (Bredel/Maaß 2017: 213) are prevented by signaling constituent boundaries with a visual structuring sign.

\section{Competing Approaches to Segmenting Com-} pounds in Easy Language

Easy Language (EL) is a rule-based variety that is adapted to the needs of people with reading impairments (for an overview of the target groups see Bredel/Maaß 2016; Rink 2020; Hansen-Schirra/Maaß 2020). Even though there is consensus in EL research that the marking of constituent boundaries decreases the time it takes for readers to locate and identify the compound's constitu- 
ents, there is no consensus on the visual structuring sign that is to be used for segmenting compounds: The first practical rulebooks of EL (Inclusion Europe 2009; BITV 2.0 2011; BMAS 2013) recommend segmenting compounds with a hyphen (Regen-Schirm [umbrella]). In spite of the lack of scientific proof and linguists' understanding that the rules are "basically assumptions based on practical experience in terms of what makes texts easier to comprehend" (Maßß 2020: 74), the rule of segmenting compounds, or more specifically "long words" (Inclusion Europe 2009: 23; BMAS 2013: 26) or "compound nouns" (BITV 2.0 2011: attachment 2, Part 2), with a hyphen was officially enshrined in law in 2011 and implemented in a wide range of texts. In Germany, it was not until 2014 that the concept of EL attracted scientific interest. Since the practical guidelines were formulated without psycholinguistic or cognitive-scientific foundation, they do not provide a sufficiently precise and scientifically based approach to translating texts into EL (cf. Maaß 2020: 78). As a result, developing a scientifically founded rulebook was a major research desideratum that was fulfilled by the Research Centre for Easy Language. In 2016, the Research Centre published the Duden Leichte Sprache, which is a "comprehensive scientific basic work" (Maaß 2020: 83) for EL (for further information see Bredel/Maaß 2016a).

The Research Centre disapproves of the intuitively based recommendation of segmenting compounds with a hyphen. Instead, it suggests structuring compounds with a hyphenation point called mediopoint (Regen-schirm). In the scientifically based rulebook, the authors elaborate numerous linguistic, social, and educational disadvantages of segmenting compounds with a hyphen, the most important of which are outlined below (for a detailed discussion see Bredel/Maßß 2016a; Мaßß 2020).

One of the most notable shortcomings of using the hyphen as a structuring sign is that segmenting compounds with a hyphen contradicts German orthography. The deliberate use of incorrect German is problematic since it discredits the EL target groups (cf. Bredel/Maaß 2016b: 9). In addition, the Research Centre for Easy Language argues that it is not only the target groups of EL that rejects texts in which words are deliberately spelled incorrectly, but also the general public (cf. Bredel/Maaß 2017; Maßß 2020). The hyphenated version, which is not only incorrect but also deviates noticeably from the standard version, therefore "triggers strong re- pulsion in readers" (Hansen-Schirra/Maaß 2020: 20). The misspelling of words also reduces the general acceptability of EL, which in turn increases the risk of stigmatizing groups that are dependent on EL to access information. Furthermore, the reader internalizes false spellings, which is problematic not only because it triggers false learning impulses but also because it makes it harder for the reader to recognize the same word beyond EL texts. Therefore, segmenting compounds with a hyphen not only contradicts one of the main principles of EL, which is to avoid incorrect spelling, but it is also incompatible with the intended learning function of EL. Another shortcoming of using the hyphen as a segmentation sign is that the uppercase character after the hyphen encourages the reader to process the compound's constituents as individual nouns (cf. Bredel/Maaß 2017: 225). While it is expected that this will not cause processing difficulties in transparent compounds, i.e., compounds "in which the meanings of each of the constituents are transparently represented in the meaning of the compound as a whole" (Libben et al. 2003: 50), it is assumed that it is counterproductive for the processing of opaque compounds, i.e., compounds whose meaning cannot be derived from the meaning of its constituents; e.g. there is no meaning of "Löwen" [Lion] or "Zahn" [tooth] in "Löwenzahn" [dandelion] (for a discussion see Deilen 2020).

Instead, marking the morpheme boundaries with a mediopoint has the following advantages: In contrast to the hyphen, the mediopoint, combined with the lowercase character after the mediopoint, complies with German orthography. Therefore, it does not negatively impact the learning function EL is intended to fulfil. Another major advantage is that, since the mediopoint is smaller and less invasive, compounds segmented with a mediopoint do not deviate from the standard version to the same extent as compounds segmented with hyphen do. Consequently, using the mediopoint as a structuring sign may allow readers to recognize the same compounds beyond EL texts. Another key advantage is that unlike the hyphen, which is a well-known punctuation mark that is already used in other contexts, such as to separate syllables or to join two names, the mediopoint is not yet used in other contexts. Even though the mediopoint is "a functional and non-stigmatising alternative to segment compound nouns" (Hansen-Schirra/Maßß 2020: 20), it still deviates from the standard version and identifies texts written in EL as such. For this reason, it still carries a certain risk of stigmatizing the target groups. Nevertheless, the correct 
spelling of words lowers the risk of committing readers to EL texts. To summarize, since the mediopoint enhances the perceptibility of the constituents without violating orthographic conventions, it is assumed that the mediopoint is more acceptable than the incorrect hyphen.

Seen from a theoretical perspective, there is little doubt that the marking of constituent boundaries facilitates access to the compound's constituents for people with low literacy skills. However, since the empirical research efforts into cognitive processing of compounds in EL have only been undertaken over the past few years, we still do not know whether or not enhanced perception of the compound's constituents also reduces cognitive processing costs. Research is needed to answer the question of whether the theoretical advantages of the mediopoint can be verified by empirical evidence. Meeting these desiderata is crucial in contributing to the development of EL, because only once the advantages of the mediopoint are verified by empirical evidence, will it be possible to reliably question the current practice of segmenting compounds with a hyphen. The question of whether the hyphen or the mediopoint can more effectively assist people with low literacy skills in accessing compounds has only been addressed in a very limited number of studies to date, which we discuss next.

Empirical Research on Compound Processing in Easy Language (and beyond)

Considering the discussion about the pros and cons of hyphen and mediopoint, Wellmann (2020) conducted an eye-tracking study with women learning German as a second language. In a word-picture-matching test, they were presented with compounds, either segmented with a hyphen, segmented with a mediopoint or not segmented at all, and were asked to match them to one of the pictures while their eye movements were recorded. Her results confirmed that compounds with a mediopoint are processed faster than compounds that are either separated with a hyphen or not optically structured at all. Furthermore, the participants made fewer mistakes if words were separated with a mediopoint. The study can therefore be seen as empirical evidence in favor of the mediopoint. Wellmann's finding that segmenting compounds is beneficial in helping people with low literacy skills process compounds is consistent with the results of Gutermuth (2020). Gutermuth's (2020) eye-tracking study, which was conducted with people with cognitive impairment, migrants, and senior citizens, revealed that migrants pro- cessed compounds with mediopoint significantly faster than unsegmented compounds. For the other target groups, the mediopoint was only beneficial during the initial phase of compound reading. Another main finding was that even though senior citizens comprehended and recalled texts in EL better than less comprehensibilityenhanced texts, the eye-tracking data revealed that there was no difference between the total reading time for unsegmented compounds and compounds segmented with a mediopoint, which indicates that processing unsegmented compounds does not cause any difficulties for senior citizens. It thus seems that segmenting compounds is only beneficial for some of the target groups of EL. In addition, Pappert/Bock (2020) conducted a study with adults with intellectual disability and adults with functional illiteracy. Their lexical decision task, in which transparent and opaque compounds were either presented with a hyphen or not optically structured at all, revealed that for EL target groups segmentation of compounds with a hyphen eases lexical access to both transparent and opaque compounds. This outcome approves segmenting compounds irrespective of semantic transparency.

The studies by Wellmann (2020), Gutermuth (2020), and Pappert/Bock (2020) only investigated the cognitive processing of compounds with two constituents. Yet, following the line of reasoning in Bredel/Maaß (2016, 2017), the difficulties when processing compounds are mainly due to their complexity and length and the obstacle to overcome rises with increasing word length. One of the reasons why polymorphemic compounds cause more difficulties is that "with each new recursive step, the compound becomes more ad hoc, more syntactic, less lexicalized, $[\ldots]$ more difficult to interpret, memorize and produce, more dependent on its context, more limited in its meaning and use and more restricted to the written language" (Berg 2006: 198). Nonetheless, studies on the processing of compounds with three or more constituents are still lacking, so that the rule of segmenting especially multimorphemic compounds with a mediopoint still awaits empirical testing. This desideratum constitutes the starting point for this study. Before presenting our experiments, we will first summarize some crucial findings of an eye-tracking study by Inhoff et al. (2000), which are of central importance to our hypotheses.

Even beyond EL research, the processing of polymorphemic compounds has received only little attention. The only eye-tracking study with unimpaired readers that has 
dealt with the question of how German compounds with more than two morphemes are processed was conducted by Inhoff et al. (2000). Inhoff et al. (2000) presented triconstituent compounds in three different boundary conditions: either concatenated (Einzelhandelsumsatz [retail sales]), with interword spaces between word boundaries (Einzel handels umsatz) or concatenated with the first letter of the constituents being marked by uppercase characters (EinzelHandelsUmsatz). While the first condition conformed to spelling conventions, the other two conditions violated spelling conventions. The study revealed that compounds with interword spaces between word boundaries were read faster than the other two conditions. Yet, unlike the first and second fixation, the final fixation on the compound was longest in the spaced condition, which indicated that compounds with interword spaces were disadvantaged in the final stage of processing. Interword spacing thus benefited the initial phase of compound reading as it facilitates locating and accessing the compound's constituents. At the same time, however, the insertion of interword spaces deprives readers from the marking of the compound's end, which hampers the identification of the constituents as a unified lexical unit. Hence, there is no visual cue indicating whether the constituent (a) is the compound's head, (b) is a part of the compound that modifies the head or, (c) already forms part of the following word. The standard and uppercase condition instead yielded "parallel results in the majority of comparisons" (Inhoff et al. 2000: 45). They conclude that not only the morpheme boundaries but also the compound's end needs to be clearly marked to facilitate cognitive processing. However, since the study was only conducted with unimpaired readers, it does not allow for valid conclusions about the processing of compounds in people with reading impairments. Still, when applying the findings of Inhoff et al. (2000) to segmenting compounds with a mediopoint, it becomes evident that using the mediopoint as a structuring sign offers the advantage of marking the morpheme boundaries without violating spelling conventions, while at the same time indicating that the constituents belong to a conceptually unified compound (cf. Bertram et al. 2011).

The eye-tracking study presented here sets out to determine which method of segmentation (the use of the hyphen or the use of the mediopoint) is better suited to assisting individuals with low literacy skills in accessing compounds. In addition, we investigate whether reading compounds without context differs from reading com- pounds with context. Based on the aforementioned studies and findings we hypothesize that segmenting compounds is beneficial in helping readers with low literacy skills process compounds, with the processing benefit being greater for compounds segmented with a mediopoint than for compounds segmented with a hyphen. Following the line of reasoning in Maaß (2015), we assume that the more morphemes the compound consists of, the greater the facilitating effect of segmenting compounds. Since unimpaired readers are used to reading unsegmented compounds, we further assume that they do not benefit from segmentation of compounds. However, considering the theoretical advantages of the mediopoint we still expect that not only impaired but also unimpaired readers process compounds segmented with a mediopoint faster than compounds segmented with an orthographically incorrect hyphen. For both impaired and unimpaired speakers we also hypothesize that the context facilitates lexical access to, and processing of, compounds.

\section{Methods}

\section{Experiment 1}

\section{Material and study design}

An initial list of 66 preselected compounds were presented to 25 undergraduate students, none of whom participated in the subsequent experiments. The students were asked to rate the familiarity and degree of abstractness of each compound. A seven-point Likert scale was employed ranging from "very low (1)" to "very high" (7). Based on the results of the rating study, we selected a set of equally familiar and equally concrete compounds. We chose 27 noun-noun compounds, nine of which contained two, three, and four morphemes. Compounds and morphemes were balanced for frequency and length. In addition, compounds were controlled for number of syllables, familiarity, and degree of abstractness.

The first eye-tracking-experiment was designed as a word-picture-matching test. Compounds were presented either concatenated or in one of two conditions in which morpheme boundaries were marked by structuring signs (i.e., hyphen or mediopoint). At the outset of the experiment, participants were informed that they would see words in the center of the screen while their eye movements were recorded. They were instructed to read the word silently. The word remained on the screen until the 
participants clicked the mouse button. Subsequently, the word was replaced by a picture slide consisting of three pictures and a question mark. Participants were asked to match the word to the appropriate picture or, in case they did not know the word, to click on the question mark. While one of the three pictures showed the content of the compound, the other two pictures showed the content of the compound's immediate constituents (see Fig. 1).

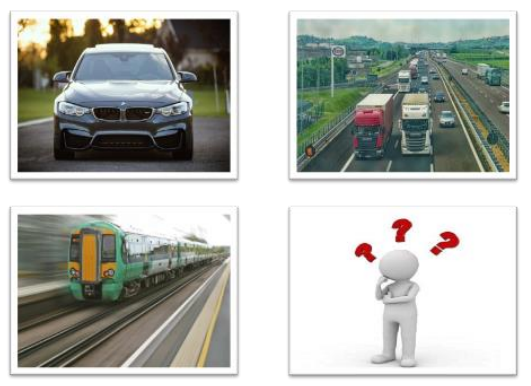

Fig. 1: Example of the poststimulus distractor task

The word-picture-matching task served as a poststimulus distractor task, which was not only used to distract the readers and to check comprehension but also to "give participants a clear purpose for reading the stimuli so that they pay attention to them for the duration of the experimental session" (Keating/Jegerski 2015). In addition to the 27 compounds presented in one of the three boundary conditions, 43 monomorphemic words with a varying number of syllables were used as distractor items. Compounds and distractors were presented in a random order. After six practice trials the main experiment was run in a single block of 70 trials.

\section{Participants}

Since so far very little is known about how compounds segmented with mediopoint are processed by unimpaired readers, our study was conducted not only with people with low literacy skills (referred to as the target group) but also with unimpaired readers. Conducting the experiments with unimpaired readers not only allowed us to infer differences in the cognitive processing of differently segmented compounds in unimpaired readers but also allowed us to compare the reading behavior of impaired and unimpaired readers. Therefore, the experiment was first conducted with 48 unimpaired students. All students were native speakers of German. They received monetary compensation for their participation. In addition, 19 pupils with prelingual hearing impairments or deafness were recruited for this study. They were selected to represent one of the heterogenous target groups of EL. The pupils were aged 13- to 17-years and attended a school for the deaf and hearing impaired (7th to 10 th grade). According to teacher and/or parental report, none of them had additional disabilities or special needs. Written consent was obtained from both pupils and parents. When it comes to reading proficiency, deaf and hard-of-hearing pupils are a heterogenous group, which is mainly due to varying degrees of hearing loss, different kinds of hearing aids and implants, and different amount of language exposure. Since several studies (e.g. Hennies 2009; Holt et al. 1997; Mitchell/Karchmer 2003) have documented this diversity of reading proficiency in the deaf population, we assessed pupils' reading skills via the Salzburg Reading Screening for Grades 2 to 9 (Wimmer/Mayringer 2014/2016), which is a standardized method for identifying pupils at-risk for reading disabilities. Based on the results, we divided the pupils into two subgroups: pupils with higher literacy skills $(n=9)$ and pupils with lower literacy skills $(n=9)$. Splitting the target group into two subgroups allowed us to compare reading behavior from pupils with higher literacy skills with reading behavior from pupils with lower literacy skills and to investigate how reading proficiency influences the need for compound segmentation (for details on the target group's reading proficiency see Deilen 2020).

\section{Apparatus and Procedure}

Eye fixation patterns were recorded using a mobile SMI Eye Tracker at a sampling rate of $250 \mathrm{~Hz}$. Participants were seated at a viewing distance of about $60 \mathrm{~cm}$ from the screen. Prior to the experiment, the eye-tracker was calibrated using a five-point calibration grid that extended over the screen. To this end, participants were instructed to fixate five dots that appeared sequentially on the screen. To verify the accuracy and stability of the fixation positions, the calibration procedure was followed by a validation process.

Trials in which a blink occurred on the compound were deleted. Based on the approach of Inhoff/Radach (1998), fixations of less than $50 \mathrm{~ms}$ were also deleted. In addition, we only included participants with a tracking ratio of at least $80 \%$. Altogether, these criteria led to the exclusion of $14.04 \%$ of the data. 


\section{Data analyses}

The following eye movement measures were calculated for analysis: number of fixations, first fixation duration, total reading time, and rate of regressions. For analyzing the eye-tracking data, we fitted linear mixedeffects models (LMMs) with fixed and random effects using the package lme4 (Bates et al. 2019). Both items and participants were considered as random effects (Baayen 2008). Given the high number of possible model specifications and with that potentially occurring convergence problems, random effects were reduced, if necessary, to reach convergence (Matuschek et al. 2017). For model fitting we used the Restricted Maximum Likelihood Method to obtain unbiased estimates for fixed and random effects (cf. Fahrmeir et al. ${ }^{2} 2009$ ). To select among candidate models with and without interaction terms, Akaike's information criterion (AIC) was used. By removing random effects that did not significantly increase the model goodness-of-fit and by choosing the model with the lowest AIC, we aimed to obtain a model structure that adequately describes the data while not being overly complex. For each dependent variable, a model was fitted containing the fixed main effects of "segmentation" (standard, hyphen, mediopoint), "number of morphemes" $(2,3,4)$ and, if selected by AIC, their interactions. The significance level for statistical tests was selected as $\alpha=.05$, where the Bonferroni correction was used to reduce the likelihood of a Type I error. Posthoc contrasts were computed using the $\mathrm{R}$ package effects (Fox 2020). We fitted separate LMMs for unimpaired readers and the target group of EL. Given the heterogeneity of pupils with hearing impairments, we subsequently fitted separate LMMs for pupils with higher literacy skills and pupils with lower literacy skills, respectively.

Descriptive statistics of the data are reported in Table 1 (unimpaired readers), Table 2 (target group), Table 3 (pupils with lower reading skills), and Table 4 (pupils with higher reading skills) in the appendix.

\section{Results}

In the following we summarize the results of Experiment 1. Further details and non-significant differences are provided in the appendix.

\section{Unimpaired readers}

Analysis revealed a main effect of segmentation $(\beta=$ $0.539, t=-2.705, p=0.007)$ with unsegmented compounds being, ceteris paribus, fixated significantly less often than compounds segmented with a mediopoint. The interaction of three morphemes and hyphenation $(\beta=$ 11.773, $t=4.178, p<0.001)$ as well as the interaction between three morphemes and concatenation $(\beta=0.582, t$ $=2.064, p=0.039$ ) was significant, indicating that trimorphemic compounds with mediopoint were read with significantly less fixations than trimorphemic compounds with hyphen. The interaction between boundary condition and number of morphemes is depicted in Fig. 2. Bimorphemic unsegmented compounds were read with less fixations than bimorphemic compounds segmented with either hyphen or mediopoint (see Fig. 2). Four-member compounds, however, were read with mediopoint with more fixations than with hyphen or without any structuring sign; however, the differences were not significant (see Appendix 2). In addition, unsegmented compounds were read with significantly less fixations than hyphenated compounds $(\beta=0.402, t=3.493, p=0.002$ ), with the difference being only highly significant for compounds with three morphemes $(\beta=-0.809, \mathrm{t}=-4.057, p=0.002)$.

However, following the line of reasoning in Wolfer (2016), "if no effect can be shown, this does not mean that there really is no effect" (Wolfer 2016: 179), rather, it means that the investigated sample is insufficient for demonstrating its significance. The fact that we "simply cannot detect the effect" (ibid.) is especially due to the conservative Bonferroni correction, which was used to reduce the chances of type I errors (i.e., the chances of obtaining false-positive results). This is achieved by lowering the alpha value to account for the number of comparisons being performed on a single data set. However, when performing a high number of comparisons, the conservative Bonferroni correction increases the risk of generating false negatives (type II errors). This in turn means that, due to the conservative Bonferroni correction, even strong tendencies, which are clearly present in our sample, may not be significant (cf. Bland/Altman 1995; Napierala 2012). Since up to 66 tests were performed on our data set and our sample was relatively small, the significance was unable to withstand the Bonferroni correction, even though strong tendencies were undoubtedly present. 
Analysis of the rate of regression showed a significant effect of hyphenation with the rate of regressions being significantly higher for compounds with a hyphen than for compounds with a mediopoint $(\beta=3.258, t=2.637, p$ $=0.008)$. Unsegmented compounds were also read with less regressions than hyphenated compounds.

\section{[Figure 2]}

No main effects of boundary conditions or interactions were found for first fixation duration. However, for compounds with two morphemes the first fixation was marginally significantly longer than for compounds with four morphemes $(\beta=20.168, t=1.790, p=0.074)$. Posthoc contrasts showed that no matter the number of morphemes, first fixation durations decreased when hyphens were inserted between constituents (see Fig. 2). Taken compounds with two, three, and four morphemes together, the first fixation for hyphenated compounds was marginally significant shorter than for unsegmented compounds $(\beta=-14.11, t=-2.173, p=0.090)$.

The LMM on total reading time yielded a significant main effect of hyphenation $(\beta=-171.76, t=-3.156, p=$ $0.002)$ and concatenation $(\beta=-125.21, t=-2.301, p=$ 0,022 ) and significant interactions of hyphenation and compounds with two and three morphemes, indicating that the, ceteris paribus, significantly shorter reading time for hyphenated and unsegmented compounds was confined to compounds with four morphemes. The significant interaction of hyphenation and compounds with three morphemes revealed that trimorphemic compounds with mediopoint are read significantly faster than trimorphemic compounds with hyphen $(\beta=177.542, t=3.262$, $p=0.041)$. This interaction is depicted in Fig. 2 .

\section{Target group}

The effect estimates indicate that unsegmented compounds were read with less fixations than compounds segmented with a mediopoint, whereas hyphenated compounds were read with more fixations than compounds segmented with a mediopoint. The effects, however, were not significant. Analysis showed no significant main effect of segmentation on the rate of regression either. However, for trimorphemic compounds with a mediopoint the rate of regression was significantly lower than for trimorphemic compounds with a hyphen $(\beta=12.321$, $t=3.685, p=0.009)$. Fig. 3 shows that for both compounds with three and four morphemes, the target group jumped backwards least often in compounds segmented with a mediopoint.

\section{[Figure 3]}

Analysis yielded no significant main effect of the boundary condition on the first fixation duration and no significant main effect of boundary condition on the total reading time either.

Target group (pupils with lower literacy skills vs. pupils with higher literacy skills)

For pupils with lower literacy skills, the main effect of hyphenation on number of fixations indicated that hyphenated compounds were read with significantly more fixation than compounds with mediopoint $(\beta=2.037, t=$ 2.491, $p=0.013$ ). For unsegmented compounds, the number of fixations was also higher than for compounds with mediopoint, but the effect was not significant. Analysis of the number of fixations for pupils with higher literacy skills showed a significant main effect of the boundary conditions indicating that, ceteris paribus, unsegmented compounds $(\beta=-1.370, t=-3.230, p=0.001)$ and hyphenated compounds $(\beta=-0.815, t=-1.921, p=$ 0.056) are read with less fixations than compounds with mediopoint. The interaction between three morphemes and hyphenation $(\beta=1.852, t=3.086, p=0.002)$ as well as the interaction between two morphemes and concatenation $(\beta=1.296, t=2.160, p=0.032)$ and three morphemes and concatenation $\beta=1.444, t=2.407, p=$ 0.017 ) was significantly positive, indicating that the higher number of fixations for compounds with mediopoint was confined to four morphemes (see Fig. 4).

\section{[Figure 4]}

For low literacy pupils, the main effect of hyphenation on the rate of regressions $(\beta=5.119, t=1.953, p=$ 0.052 ) indicated that the rate of regression was for hyphenated compound marginally significantly higher than for compounds with mediopoint. For unsegmented compounds the rate of regression was also higher than for compounds with mediopoint, however, the effect was not significant. Fig. 4 shows that irrespective of the number of morphemes, pupils jump backwards most often when compounds are hyphenated. For pupils with higher literacy skills, analysis did not yield a significant main effect of boundary conditions, still the effect estimates indicate that both hyphenated and unsegmented compounds elicit a higher rate of regressions than compounds with medi- 
opoint. The interaction between two morphemes and hyphenation $(\beta=-12.333, t=-1.835, p=0.068)$ and three morphemes and hyphenation $(\beta=17.889, t=2.661, p=$ $0.008)$ revealed that the higher rate of regression for hyphenated compounds is confined to three and four morphemes (see Fig. 4).

For pupils with lower literacy skills, we found a marginally significant main effect of hyphenation $(\beta=$ $373.94, t=1.832, p=0.068$ ) on total reading time, indicating that hyphenated compounds elicited longer gaze durations than compounds with mediopoint. For unsegmented compounds, the total reading time was also longer than for compounds with mediopoint, but the effect was not significant. In addition, a significant interaction between three morphemes and hyphenation emerged $(\beta=$ -742.27, $t=-2.546, p=0.012)$, indicating that the shorter reading time for compounds with mediopoint was restricted to compounds with two and four morphemes (see Fig. 4). For pupils with higher literacy skills, we found a significant main effect of hyphenation $(\beta=-258.40, t=$ 2.263, $p=0.025)$ and concatenation $(\beta=-305.27, t=-$ $2.674, p=0.008$ ) on total reading time as well as significant interactions between three morphemes and hyphenation $(\beta=471.63, t=2.921, p=0.004)$, three morphemes and concatenation $(\beta=420.19, t=2.603, p=0.010)$ and a marginally significant interaction between two morphemes and concatenation $(\beta=309.20, t=1.915, p=$ 0.057). The interactions reveal that the facilitatory effects of hyphenation and concatenation are confined to compounds with two and four morphemes (see Fig. 4). Trimorphemic compounds with mediopoint, in turn, elicit shorter reading times than trimorphemic concatenated and hyphenated compounds.

Furthermore, we found significant differences between pupils classified as having low reading skills and pupils classified as having higher reading skills, which not only backs up separating the target group into two groups, but also confirms the findings of previous eyetracking studies (e.g., Häikiö et al. 2011; Hasenäcker et al. 2017). Firstly, less-skilled pupils read compounds with significantly (and on average 58.3\%) more fixations than pupils with more advanced reading skills $(z=-8.637, p<$ 0.001, $r=0.393$ ). Secondly, they jumped backwards significantly (and on average 12.1\%) more often than better-skilled pupils $(z=-2.131, p=0.033, r=0.097)$ and read the compounds significantly (and on average 64.9\%) slower than better-skilled pupils $(z=-8.539, p<0.001, r$
$=0.389)$. Lastly, the first fixation duration differed significantly between less-skilled and better-skilled pupils $(z=$ -3.182, $p<0.001, r=0.145)$, with the first fixation being on average $13 \%$ shorter for less-skilled pupils than for better-skilled pupils.

The fact that the first fixation duration, which represents early processing stages, was significantly shorter for less-skilled readers than it was for higher-skilled readers was at first surprising, especially because all other parameters indicated that cognitive processing of compounds was easier for pupils with stronger reading skills. This finding of a shorter first fixation for less-skilled readers was confirmed when comparing the first fixation for unimpaired readers and the target group, which revealed that the first fixation of the target group was significantly shorter than the first fixation of unimpaired readers $(z=-2.963, p=0.003, r=0.071)$. This finding could suggest a short first fixation might not necessarily be indicative of a faster processing of compounds. We will return to this assumption in the discussion, where we also provide a possible explanation for it. The question of whether our findings regarding the first fixation converge with other studies on the cognitive processing of compounds is addressed in the discussion as well.

\section{Discussion}

With the first experiment we aimed to determine whether reducing compounds' complexity by inserting a segmentation sign has a facilitating effect on the processing of compounds. Our results demonstrated that segmentation of compounds disrupts reading among unimpaired readers. However, it seems that they process compounds with mediopoint faster than compounds with hyphen. Even though most of the results of the target group revealed as well that, overall, compounds with mediopoint are processed more easily than compounds with hyphen, we also found some discrepancies. In addition, the data did not allow us to answer the question of whether unsegmented compounds or compounds segmented with mediopoint were processed better, and thus the question of whether segmenting compounds is at all necessary to facilitate processing of compounds. Since these discrepancies are in all probability at least partly due to the pupils' heterogenous reading skills, it was necessary to take a closer look at the eye-tracking data of low-skilled and high-skilled pupils, respectively. By doing so, analysis revealed that for individuals with low literacy skills reducing linguistic complexity by marking 
constituents' boundaries helped to reduce cognitive processing costs. In addition, we could prove that low-skilled readers generally processed compounds with a mediopoint faster than compounds with a hyphen. Since none of the participants asked for the meaning of the mediopoint - a sign which was in all probability unknown to them - we can deduce that even readers with low literacy skills understand the mediopoint intuitively (cf. Bredel/Maaß 2017: 218 ff.) - which is an indispensable requirement for using it as a segmentation sign.

Furthermore, we found evidence that lower-skilled pupils especially profit from visual segmentation in fourmember compounds. So far, we can thus conclude that structuring signs facilitate decomposition and help individuals with low literacy skills to process the word complex in small chunks (cf. Bredel/Maaß 2017: 221). On the contrary, our data did not indicate that pupils with higher skills profited from segmented word forms. Only for trimorphemic compounds did we find a distinct processing advantage for compounds with mediopoint over unsegmented compounds. For compounds with two and four morphemes, however, we could neither prove a processing advantage over hyphenated compounds nor a processing advantage over unsegmented compounds.

Our results converge with the findings of Pappert/Bock (2020) in that they also indicate that readers with very low reading skills profit from visual segmentation of compounds. In addition, both studies have shown that unimpaired readers process unsegmented compounds better than segmented compounds. Our results are also consistent with the findings of Wellmann (2020), who showed that compounds with mediopoint are processed better than compounds with hyphen.

All in all, we could mostly confirm the hypothesis, that the target group profits from segmentation with the mediopoint, and we could also prove that the need for segmentation depends on recipients' reading proficiency. However, since the experiment on word level also yielded some contradicting results, which did not confirm the hypotheses, and partly also indicated a processing advantage of the hyphen, we are still unable to rule out that also the hyphen yields some processing advantage.

So far, we are also unable to answer the question of whether our findings converge with previous studies on the processing of compounds. The reason for this is that in most studies (except for the studies by Wellmann
(2020) and Pappert/Bock (2020)) compounds were not presented isolated but embedded in sentences. Since there is overwhelming evidence that reading words embedded in context differs considerably from reading and recognizing the same words presented without context, we cannot assume that our findings generalize to normal reading. One of the main reasons for this is that sentence reading is a more dynamic and more natural "task in which a sequence of words is to be processed" (Inhoff et al. 2000: 30) and in which the meaning of the compound is to be integrated in and partly assigned by the context. Since eye-tracking experiments on word and sentence level led to differing results in a wide range of studies (e.g., Vitu et al. 1990; O'Regan 1992; for an overview see Nicholson 1993), we designed a second experiment, in which we presented the same compounds again, but this time embedded in meaningful sentences. The aim of the second experiment was twofold: Firstly, we aim to investigate whether the findings on word level extend to normal reading, i.e., the effects of Experiment 1 also emerge when presenting compounds in sentences. Secondly, we address the question of whether compound processing is facilitated by contextual information, i.e., whether reading compounds in context, and with that in a less artificial and more ecologically valid situation, is easier than reading them without context. If this were the case, it would be possible that, as Pappert/Bock (2020) suggest, "context might help lexical identification and comprehension in less-skilled readers and render compound segmentation dispensable" (Pappert/Bock 2020: 1122). Embedding the same compounds in simple sentences will also serve to reveal or rule out alternative other factors that might explain the results on word level.

\section{Experiment 2}

The compounds were the same 27 compounds presented in Experiment 1, but this time they were embedded in sentences. After reading the sentence, the participants were presented with a binary choice question and were asked to decide whether the sentence they had just read was meaningful or not. By using this binary choice question as a poststimulus distractor task, we ensured that the participant not only fixated but also cognitively processed the compound. To this end, it was necessary to include additional nonsense sentences, which were supposed to generate "no" responses in the binary choice question. The sentences containing a compound accounted for 27 of the 80 sentences presented to each partici- 
pant. These 27 target sentences were mixed with 53 filler sentences, containing the same distractor items as in Experiment 1, and 10 nonsense sentences. The order of sentences was randomized. In addition to controlling the sentence length, we also ensured that the word preceding and following the target word contained at least four characters. By controlling the length of the word before and after the compound, we reduced the risk of the compound being skipped. We also controlled the compound position, with the compound occupying neither the start nor the end of a sentence. The naturalness and comprehensibility of the sentences served as additional control variables. To ensure that the sentences were on average as natural and comprehensible as possible, we asked 25 undergraduate students to rate the naturalness and comprehensibility of a larger pool of sentences, containing either a compound or a monomorphemic word, on a seven-point Likert scale. None of the students participated in the eye-tracking experiment.

Eye-tracking data were again analyzed by fitting LMMs. Model fitting was conducted in the same way as described in Experiment 1. In a next step, we included the context as an additional explanatory variable, which allowed us to compare the eye-tracking data for compounds that were read with and without context. We computed the same eye movement variables for analyses as in Experiment 1 . In addition, we tracked the number of revisits, which reports how often the compound is viewed after being left for the first time. About $13.47 \%$ of the data were excluded before analyses due to insufficient tracking ratio, skipping of the target word, blinks or fixations being shorter than $50 \mathrm{~ms}$.

Descriptive statistics of the data are reported in Table 5 (unimpaired readers), Table 6 (target group), Table 7 (pupils with lower reading skills), and Table 8 (pupils with higher reading skills) in the appendix.

\section{Results}

\section{Unimpaired readers}

The significant main effects indicated that compounds with hyphen were read with significantly more fixations than compounds with mediopoint $(\beta=0.518, t=3.704, p$ $<0.001$ ), whereas unsegmented compounds were read with significantly less fixations than compounds with mediopoint $(\beta=-0.591, t=-4.220, p<0.001)$ and hyphen $(\beta=-0.706, t=-8.665, p<0.001)$. Fig. 5, which depicts the interaction between number of morphemes and segmentation, shows that no matter the number of morphemes, the number of fixations is always lowest if the compound is unsegmented, whereas the number of fixations is always highest when compounds are segmented with hyphen.

The main effect of hyphenation on revisits indicated that hyphenated compounds were revisited marginally significantly more often than compounds with mediopoint $(\beta=0.090, t=1.865, p=0.062)$. Hyphenated compounds were also revisited more often than unsegmented compounds, the difference, however, did not withstand the Bonferroni correction. Furthermore, Fig. 5 shows that no matter the number of morphemes, hyphenated compounds clearly caused the highest ratio of regressions. Compared to unsegmented compounds, first fixation durations decreased for compounds with two, three, and four morphemes when hyphens were inserted. For the mediopoint, this was only true for trimorphemic compounds (see Appendix 2). In addition, the main effect of hyphenation on total reading time indicated that compounds with hyphen were read significantly slower than compounds with mediopoint $(\beta=130.08, t=3.508, p<$ $0.001)$. On the other hand, the main effect of concatenation indicated that unsegmented compounds were read significantly faster than compounds with mediopoint $(\beta=$ -80.91, $t=-2.178, p=0.030)$. Fig. 5 shows that no matter the number of morphemes, unsegmented compounds always yield the shortest reading time whereas hyphenated compounds always yield the longest reading time.

\section{[Figure 5]}

\section{Target group}

For the target group, the marginally significant effect of concatenation indicates that unsegmented compounds were read with less fixations than compounds with mediopoint $(\beta=-0.316, t=-1.700, p=0.090)$ and hyphen $(\beta=$ $-0.614, t=3.346, p=0.003)$. Hyphenated compounds were read with more fixations than compounds with mediopoint, however, this was only true for compounds with three and four morphemes (see Appendix 2).

The LMM on revisits did not yield a significant effect of segmentation, however, the direction of the effect estimates indicates that concatenated compounds are revisited less often than compounds with mediopoint, whereas hyphenated compounds are revisited more often than compounds with mediopoint. In terms of the ratio of regression, the positive direction of the effect estimates 
indicates that the ratio of regression is for both hyphenated and unsegmented compounds higher than for compounds with mediopoint. However, this is only true for compounds with three and four morphemes (see Fig. 6). Post-hoc contrasts also showed that for compounds with three and four morphemes, first fixation durations decreased when a segmentation sign was inserted between constituent words (see Fig. 6). However, none of the differences withstood the Bonferroni correction (see Appendix 2). The effect of segmentation on the total reading time was not significant either, however, Fig. 6 shows that no matter the number of morphemes, hyphenated compounds always yielded the longest reading time.

\section{[Figure 6]}

Target group (pupils with lower literacy skills vs. pupils with higher literacy skills)

For pupils with lower literacy skills, the main effect of hyphenation revealed that hyphenated compounds were read with significantly more fixations than compounds with mediopoint $(\beta=1.276, t=2.615, p=0.010)$. For unsegmented compounds, the number of fixations was also higher than for compounds with mediopoint, but the effect was not significant. The two morphemes $\mathrm{x}$ hyphenation interaction $(\beta=-1.561, t=-2.253, p=$ $0.025)$ revealed that, unlike compounds with three and four morphemes, bimorphemic compounds with hyphen were read with less fixations than bimorphemic compounds with mediopoint. The effect, however, did not withstand the Bonferroni correction. The interaction is depicted in Fig. 7. For pupils with higher literacy skills, the main effect of concatenation revealed that they read unsegmented compounds with significantly less fixations than compounds with mediopoint $(\beta=-0.532, t=-2.218$, $p=0.028)$. Post-hoc contrasts indicated that no matter the number of morphemes, unsegmented compounds are always read with less fixations than compounds with mediopoint or hyphen. For compounds with two and four morphemes, the number of fixations is for compounds with mediopoint lower than for compounds with hyphen, whereas for triconstituent compounds the number of fixations is for compounds with mediopoint slightly higher than for compounds with hyphen. However, none of the differences were significant (see Appendix 2).

When reading hyphenated compounds, low-skilled readers jump backwards marginally significantly more often than when reading compounds with mediopoint $(\beta$
$=9.098, t=1.880, p=0.062)$. The ratio of regression is for unsegmented compounds also higher than for compounds with mediopoint, but the effect was not significant. However, the significant interaction of two morphemes and hyphenation $(\beta=-15.495, t=-2.253, p=$ 0.025 ) revealed that the lower ratio of regression for compounds with mediopoint was confined to compounds with three and four morphemes. For pupils with higher reading skills, hyphenated compounds yielded the highest rate of regressions in compounds with two and three morphemes, whereas in compounds with four morphemes they yielded the lowest rate of regression. However, none of the differences were significant (see Appendix 2).

\section{[Figure 7]}

For low-skilled readers, the LMM on the first fixation duration did not reveal significant effects either. For high-skilled readers however, the main effect of concatenation $(\beta=68.94, t=1.878, p=0.062)$ revealed that the first fixation was for unsegmented compounds marginally significant longer than for compounds with mediopoint. Fig. 7 shows that for compounds with three and four morphemes, unsegmented compounds clearly yield the longest first fixation, whereas for bimorphemic compounds there is almost no difference between the three boundary conditions.

In addition, the effect estimates indicate that lowskilled readers read unsegmented and hyphenated compounds slower than compounds with mediopoint. Posthoc contrasts confirmed that regardless of the number of morphemes, hyphenated compounds yield the longest reading time (see Appendix 2). For higher-skilled pupils, Fig. 7 shows that unsegmented compounds yield the shortest reading time, whereas there is almost no difference between compounds with hyphen and mediopoint.

Similar to Experiment 1, we found significant differences between pupils with higher reading skills and pupils with lower reading skills, which not only backed up splitting up the target group into two groups, but which is also in line with several other studies (e.g., Häikiö et al. 2011; Hasenäcker et al. 2017). Low-skilled pupils read the compounds with marginally significantly (and on average $10.7 \%$ ) more fixations than better-skilled pupils ( $z=-1.794, p=0.073, r=0.083$ ). The different reading skills were confirmed by the significantly slower reading time $(z=-5.236, p<0.001, r=0.241)$, with less-skilled pupils reading the compounds on average $45.2 \%$ slower 
than better-skilled pupils. Unlike Experiment 1, neither the ratio of regressions nor the first fixation duration differed significantly between low- and high-skilled pupils. However, unlike Experiment 1, the first fixation was for low-skilled readers not shorter but (on average $2.1 \%$ ) longer than for high-skilled readers. The finding that the first fixation on sentence level was for less-skilled readers longer than for better-skilled readers was confirmed when comparing the first fixation duration for unimpaired readers and the target group, which revealed that the first fixation of the target group was significantly longer than the first fixation of unimpaired readers $(z=-5.934, p<$ $0.001, r=0.143)$. This tendency contradicts the finding of Experiment 1, in which the first fixation was for lessskilled readers significantly shorter than for better-skilled readers. We return to these results in the discussion.

\section{Contextual Effects}

As outlined at the beginning, we also investigated whether the context has a facilitating effect on the processing of compounds. Including the context as an additional explanatory variable in the LMM reveals that, for unimpaired readers, the context clearly has a facilitating effect on compound reading: The number of fixations was significantly lower if compounds were presented with context $(\beta=1.716, t=27.060, p<0.001)$. This was confirmed by the number of single fixations, which showed that if presented in context, compounds were read significantly more often with only one fixation than compounds presented without context $(\beta=0.164, t=$ 14.603, $p<0.001)$. In addition, presenting compounds with context led to a significant decrease in regressions $(\beta$ $=12.465, t=16.932, p<0.001)$ as well as to a significantly shorter total reading time $(\beta=440.39, t=25.410$, $p<0.001)$. For all three boundary conditions, all above mentioned effects were significant (see Appendix 3). Surprisingly, we did not find a significant effect of context on the first fixation duration.

For the target group, there is no doubt either that the context has a facilitating effect on compound reading. Analysis revealed that compounds with context were read with significantly less fixations than compounds without context $(\beta=1.981, t=13.604, p<0.001)$. Furthermore, we found a significant decrease in regressions for compounds presented with context $(\beta=10.595, t=9.287, p<$ $0.001)$. The effects were significant for all three boundary conditions (see Appendix 3). In addition, compounds presented with context were read significantly faster than compounds presented without context, however, this facilitating contextual effect was only significant for unsegmented compounds $(\beta=306.19, t=4.376, p<$ $0.001)$ and compounds with mediopoint $(\beta=260.38, t=$ $3.684, p=0.004)$. In contrast to unimpaired readers, the context also had a significant effect on the first fixation duration, with the first fixation being significantly longer if compounds were presented with context. However, the effect was only significant for unsegmented compounds $(\beta=-62.346, t=-3.937, p=0.001)$.

This facilitating effect of the context emerged for both low- and high-skilled readers. No matter the visual segmentation, the number of fixations was in both groups significantly lower if compounds were presented with context (see Appendix 3). In both groups, we also found a significant decrease in regressions for compounds presented with context. For low-skilled readers, this effect was significant for all three boundary conditions. For high-skilled readers, however, the effect was only significant for concatenated and hyphenated compounds (see Appendix 3). In addition, the first fixation was significantly longer if compounds were presented with context (low-skilled pupils: $\beta=63.240, t=4.503, p<0.001$, high-skilled pupils: $\beta=33.584, t=2.833, p=0.005$ ). For low-skilled readers, the effect was marginally significant for concatenated and hyphenated compounds, whereas for high-skilled pupils the effect was only marginally significant for unsegmented compounds (see Appendix 3 ). In both groups, we also found a significantly shorter reading time for compounds presented with context (lowskilled pupils: $\beta=-370.51, t=-5.500, p<0.001$, highskilled pupils: $\beta=-110.12, t=-2.537, p=0.012$ ). However, when looking at the effects for the boundary conditions separately, the effect was only significant in lowerskilled pupils for unsegmented compounds and compounds with mediopoint (see Appendix 3).

\section{Discussion}

The second experiment sought to determine whether segmenting compounds on sentence level facilitates processing. For unimpaired readers, the eye-tracking data revealed that they do not profit from segmentation since, as in Experiment 1, they process unsegmented compounds faster than segmented compounds. Analogously to Experiment 1, the data on sentence level confirmed as well that compounds with hyphen are processed significantly slower than compounds with mediopoint. Furthermore, we found evidence that no matter the visual 
segmentation, both unimpaired readers and low- and high-skilled readers of the target group process compounds significantly faster if presented with contextual information. Overall, analysis also revealed that, just like unimpaired readers, the target group processes hyphenated compounds slower than unsegmented compounds. Similar to unimpaired readers, the target groups' eyetracking data also revealed that processing compounds with a hyphen was more effortful than processing compounds with a mediopoint. When looking at the target group as a whole, however, we were not able to answer the question of whether unsegmented compounds or compounds with mediopoint were processed faster and consequently the question of whether segmenting compounds is at all necessary to facilitate processing of compounds in sentences. To answer the question of whether the use of a segmentation sign is redundant it was therefore beneficial to take a closer look at the data of lowskilled and high-skilled pupils, respectively. For lowskilled pupils, the data confirmed the conclusion drawn from Experiment 1: Not only on word but also on sentence level did low-skilled pupils profit from segmentation, however, this processing advantage only became clearly visible for compounds segmented with mediopoint. Using the hyphen, on the other hand, did not lead to verifiable processing benefits for low-skilled pupils.

We can conclude that the mediopoint is an effective way to facilitate cognitive processing of compounds. In addition, our eye-tracking results support the assumption underlying the rule for segmenting compounds in EL that reading long complex words requires much more cognitive effort than reading short words. We can thus conclude that not only from a theoretical but also from a empirical perspective does segmenting compounds with mediopoint offer more advantages than segmenting compounds with an orthographically incorrect hyphen. At the beginning of this section (page $\mathrm{xx}$ ), we mentioned that, due to the presumed facilitating effect of the context, it might be possible that, as Pappert/Bock (2020) suggest "context might help lexical identification and comprehension in less-skilled readers and render compound segmentation dispensable" (Pappert/Bock 2020: 1122). However, since our findings revealed that low-skilled readers (which are the only participants who profited from segmentation) also profited from segmentation when reading compounds in context, we could prove that, for our study, this assumption turns out to be incorrect. The data of pupils with higher literacy skills confirm the findings on word level, i.e., it seems that they do not have any problems with processing unsegmented compounds, which in turn means that they do not profit from segmentation. In this regard it seems to be true that segmenting compounds is dispensable for some of the EL target groups, i.e., those with relatively high reading skills. However, analogously to Experiment 1, the opposed tendencies do not allow us to conclude whether for higher-skilled pupils the processing effort is lower for compounds with hyphen or for compounds with mediopoint.

\section{Additional Findings}

Analyses of unimpaired readers' first fixation duration yielded some unexpected results. While in both experiments, the number of fixations, the ratio of regressions and the total reading time indicated a processing advantage for unsegmented compounds, first fixations on compounds showed a marginally significant advantage for hyphenated compounds. The finding that hyphenated compounds received longer gaze durations but shorter first fixations, which at first might seem contradicting, is consistent with several other eye-tracking studies on the processing of compounds. For example, the studies of Juhasz et al. (2005), Häikiö et al. (2011), Bertram/Hyönä (2013), and Placke (2001) have shown that the insertion of segmentation cues decreased the duration of a compound's initial fixation but increased the duration of a compound's overall gaze duration. This initially surprising short first fixation duration for segmented compounds (especially for hyphenated compounds) also converges with eye-tracking studies by O'Regan et al. (1984), Vitu et al. (1990, 1995), and Rayner et al. (1996), which highlighted that first fixation duration was longer when it landed near the optimal landing position (word center) than when it landed further from the optimal landing position. When landing near the optimal landing position, the compound can already be recognized during the first fixation, which significantly reduces the need for the reader to refixate the compound and significantly decreases the total reading time, compared to compounds in which the first fixation lands on a less optimal landing position for word identification, i.e., further to the left.

By running post-hoc analysis, we were able to confirm this proposed relationship between initial landing position and decomposed and direct access, respectively: When reading hyphenated compounds, the eyes' initial landing position was displaced more toward the beginning of the compound than it was for unsegmented com- 
pounds $(\beta=-5.07, t=-0.844, p=1.000)$. This result was supported by the significant correlation between initial landing position and first fixation $(r=0.190, p<0.001)$, which indicates that the further the first fixation lands to the left, the shorter the initial fixation duration. If, on the other hand, the first fixation landed further into the compound, as it is the case for unsegmented compounds, it is longer and thus indicative of holistic processing. This relationship between initial landing position and compositional or holistic processing, respectively, could also be proven for the target group: When reading hyphenated compounds, the eyes' initial landing position was shifted to the left, compared to reading unsegmented compounds $(\beta=-11.83, t=-0.728, p=1.000)$ and compounds with mediopoint $(\beta=-14.21, t=-0.858, p=1.000)$. In addition, the initial landing position correlated significantly with first fixation duration $(r=0.133, p=0.004)$, indicating that the further the first fixation lands to the left, the shorter the initial fixation duration. Therefore, we can infer that the hyphen affects the initial landing position and restricts the readers' attentional focus to the first constituent. In contrast, when reading unsegmented compounds, readers tend to direct the eyes toward the word's center, which is regarded as the optimal location for its recognition (cf. O'Regan/Lévy-Schoen 1987; Vitu et al. 1990). Therefore, their attention is stretched over the whole word, which enables direct access to the wholeword representation. Consequently, the longer first fixation indicates that the reader's eyes land further into the word, which enables them to directly process the compound as a whole. The fact that the readers do not only process the first morpheme, but instead the whole compound, also results in less refixations. Our results provide therefore substantial evidence that hyphenation enforces a compositional processing.

In hyphenated compounds the reader tends to land on the first constituent and their attention is at first restricted to a shorter and more frequent unit, which leads to initial processing advantages (i.e., a shorter first fixation) for hyphenated compounds. However, the subsequent processing stages are "disrupted by the presence of a hyphen at the constituent boundary, presumably due to encouraging sequential processing in case where simultaneous constituent processing and with that rapid access to whole-word representation is a viable option" (Bertram/Hyönä 2013: 161).
Following the line of reasoning in the aforementioned studies, we therefore argue that hyphenation of compounds is beneficial for lexical decomposition and for accessing the compound's first constituent, which is reflected by the shorter first fixation, compared to concatenated compounds. However, as the total reading time and the number of fixations reflect, the enforced focusing on the first constituent only requires additional processing time and is detrimental for accessing the compound via the more rapid and usually preferred direct route. Instead of accessing the whole-word representation, the reader has to access (at least) two representations and is subsequently required to assemble the individual words together, which requires additions processing efforts (cf. Bertram/Hyönä 2013). Therefore, the hyphen is a useful segmentation cue in that it signals the reader parafoveally where one constituent ends and another begins. One of the downsides of the hyphen, however, is that it enforces the reader to limit his attention predominantly to the first constituent, even though without the hyphen the reader would be capable of simultaneously extracting and processing visual information of the following constituents (cf. Bertram/Hyönä 2013). Concatenated compounds instead allow for quick activation of the whole compound.

Against this background, we are now also able to explain why the first fixation in Experiment 1 is significantly longer for participants with higher literacy skills than for participants with lower literacy skills, Considering that unimpaired readers read the compounds significantly faster and with significantly less fixations than the target group, the result that the first fixation duration increases with increasing reading proficiently was at first surprising. However, given the theoretical framework and findings of previous studies, it has now become apparent that a longer first fixation, combined with a shorter total reading time and a lower number of fixations, indicates that the compound is accessed directly by its whole-word representation. The significant differences between the groups therefore allow for the conclusion that, in contrast to impaired readers, unimpaired readers process compounds primarily via the whole-word representation, which is reflected by a longer first fixation, a shorter reading time, and lower number of fixations. However, the previous theoretical explanations have clearly demonstrated that the hyphenation of compounds is detrimental for its direct access, which in turn would imply that both impaired and unimpaired readers access hyphenated 
compounds via morphological decomposition. But if both groups process the compound via the morpheme-based route, how is it possible that the first fixation is significantly longer in readers with higher reading skills? To answer this question, we looked at the differences for the three boundary conditions separately. By doing so, it became apparent that the difference in first fixations was significant for concatenated compounds $(z=-2.587, p=$ $0.010, r=0.107)$ and marginally significant for compounds with mediopoint $(z=-1.770, p=0.077, r=$ 0.073). However, for hyphenated compounds the unimpaired readers' first fixation was only minimal and not significantly longer than the target groups' first fixation $(z=-0.757, p=0.449, r=0.031)$, which supports the assumption that both impaired and unimpaired readers process hyphenated compounds via morphological decomposition and that hyphenation is detrimental to accessing the compound via the direct route.

When presenting compounds in sentences, a different pattern emerged. Here, the first fixation was, in contrast to Experiment 1, for less-skilled readers longer than for high-skilled readers. This tendency emerged not only when comparing the first fixation duration within the target group (the first fixation for less-skilled readers was on average $2.1 \%$ longer than for better-skilled readers of the target group), but also when comparing the first fixation duration for unimpaired readers and the target group, which revealed that the first fixation of the target group was significantly and on average $11.4 \%$ longer than the first fixation of unimpaired readers $(z=-5.934, p<0.001$, $r=0.143$ ). As noted before, this tendency contradicts the finding of Experiment 1, in which the first fixation was for less-skilled readers significantly shorter than for better-skilled readers, which was taken as an indication of a morpheme-based processing of the compound. However, as shown above, a significantly shorter first fixation is only indicative of a morpheme-based access if it appears in combination with a significant higher number of fixations and a significant longer total reading time. Since the unimpaired readers read the compounds with significant less fixations and significantly faster, the significant shorter first fixation can, in this experiment, not be regarded as evidence for a morpheme-based processing of the compound. Instead, the results indicate that, in combination with a significant lower number of fixations and a significant shorter reading time, a significant shorter first fixation indicates that if compounds are presented in sentences, unimpaired readers process compounds signif- icantly faster than impaired readers at all processing stages. We assume that, in addition to their higher reading skills and experiences, this unrestricted processing benefit for readers with higher reading skills when reading compounds in context is also due to the fact that readers with higher reading skills have a larger inventory of frames at their disposal, which in turn enables them to recognize and process the words faster than less experienced readers (see also Bredel/Maaß 2016a).

\section{Discussion}

In German Easy Language, four separate guidelines postulate that compounds are to be optically segmented with either hyphen or mediopoint to facilitate lexical access to the compound's constituents. However, empirical research efforts into cognitive processing of compounds in EL are still lacking. To address this research gap, we conducted two eye-tracking experiments evaluating the effect of visual segmentation (hyphen and mediopoint), number of morphemes, and context on the processing on compounds in unimpaired readers and in deafand hard-of-hearing pupils. The study demonstrated that the benefit of segmentation is dependent on reading proficiency. While less-skilled readers of the EL target group clearly benefited from segmentation of compounds, it seems that for deaf- and hard-of-hearing students with higher literacy skills, segmentation of compounds is unnecessary. Furthermore, we showed that the marking of morpheme boundaries with a mediopoint is an effective means of reducing cognitive processing costs for individuals with low literacy skills. Moreover, we provided substantial empirical evidence that segmenting compounds with mediopoint offers more processing advantages than segmenting compounds with an orthographically incorrect hyphen - at least for unimpaired readers and low-skilled pupils with hearing impairment. Furthermore, our results indicate that reading compounds without context differs from reading compounds with context and that the context facilitates the processing of compounds regardless of readers' reading proficiency.

Our findings support the assumption of Pappert/Bock (2020) that "the need for compound segmentation will interact with reading proficiency" (Pappert/Bock 2020: 1123). The result that unimpaired readers do not benefit from segmentation signs also converges with the study by Pappert/Bock (2020), which demonstrated that segmenta- 
tion of compounds disrupts the reading process for unimpaired readers (cf. ibid.: 1110). We were able to explain this result by showing that the hyphen forces a morpheme-based access and is therefore detrimental for the holistic processing of the compound. Consequently, the hyphen is a useful segmentation cue in that it enables fast recognition of the compound's first constituent. However, since the hyphen restricts the readers' attentional focus to the first constituent and enforces sequential processing of the compounds' constituents, it hinders them from accessing the compound via the direct route and consequently slows down the processing of the whole compound word. Concatenated compounds instead are usually processed via the more rapid direct route, in which the whole-word representation of the compound is activated.

Our pattern of results can best be explained by hybrid models of compound processing, as they are proposed by Schreuder/Baayen (1995), Libben (2006), or Kuperman et al. (2009). It seems that when processing compounds, both constituent and whole-word information can be activated, which implies that the morpheme-based route competes with the direct look-up of the compound. Since less-skilled readers usually process compounds via the morpheme-based route, they are more dependent on visual cues marking the morpheme boundaries. Readers with higher reading skills predominantly process compounds in a holistic fashion, thus making parallel use of all constituents available in the foveal and parafoveal span.

Our findings for unimpaired readers, however, partly stand in contrast to eye-tracking findings by Bertram/Hyönä (2003) and Bertram/Hyönä (2013), who demonstrated that adult readers benefit from hyphenation in long compounds. This finding is explained by the fact that long compounds do not fully fit in the foveal area, where visual acuity is at its best. Therefore, when reading long compounds, adult readers often resort to the decomposition route, starting by identifying and processing the first constituent. Since hyphenation facilitates the identification of the first and all other constituents, adult readers benefit from visual segmentation in long compounds, in which they initially access the first constituent anyway. However, we were unable to reliably show a facilitating effect of hyphenation in long compounds for unimpaired readers. These differences may be accounted for by language-specific characteristics since none of the studies were conducted with German compounds. As Häikiö et al. (2009) suggest, "it may well be that the size of specif- ic components of the perceptual span are language specific (Rayner, 1998)" (Häikiö et al. 2009: 171) and that the development and use of the letter identity span differs between languages. So far, we still do not know whether unimpaired readers process long German compounds which extend beyond foveal vision via the decompositional or the holistic route. However, we do know that, even if processed via decomposition, unimpaired readers do not profit from hyphenation. This might be due to the fact that they are more familiar with reading concatenated long compounds than hyphenated long compounds and when reading hyphenated compounds, they presumably only expect a hyphen at the major morpheme boundary (as it is used in the word "Mehrzweck-Küchenmaschine") and not between every constituent. Therefore, they are disrupted by the presence of hyphens at every morpheme boundary (Mehr-Zweck-Küchen-Maschine).

Furthermore, we want to emphasize that our study only makes a small contribution to the debate of segmenting compounds in EL and that it is important to locate our results in the bigger picture. In particular, this means that there are several other factors and questions that need to be considered when arguing for the hyphen or mediopoint, respectively - factors and questions we were unable to pursue in our study. For example, we still have to investigate the extent to which other linguistic features, such as the word's opacity or linking elements, influence the need for segmentation of compounds (for first results see Deilen 2020). Furthermore, acceptability also plays an important role in the reading process (see HansenSchirra/Maaß 2020). So even though compounds might be processed better if segmented, it is also important that the mediopoint is accepted not only by the general public but also by the target groups, which we still do not know.

Our finding that recipients from the target group with higher literacy skills do not seem to profit from segmentation converges with findings from previous eyetracking studies on the processing of compounds (e.g., Gutermuth 2020; Häikiö et al. 2011). Following the line of reasoning in Gutermuth (2020) and Maaß (2020) we suggest that this missing processing benefit for recipients from the target group with higher literacy skills can be explained by the process-based capacity model for EL proposed by Gutermuth (2020) and with that, by the interaction of linguistic levels. We presented compounds only isolated or within simple sentences, which implies that the complexity at the phrasal, syntactic, and textual 
level was maximally reduced. Reducing the complexity at three out of four linguistic levels to a minimum might relieve the overall capacity, meaning more capacities are available that can be used for the processing of complex morphological structures (i.e., unsegmented compounds). Therefore, our findings support the framework proposed by Gutermuth (2020), who stated that the easier the texts, the more capacity remains for the processing of unsegmented compounds. Based on the proposal of Gutermuth (2020), it is possible that, for recipients with higher literacy skills, segmentation of compounds is unnecessary if compounds are embedded in a text, which is characterized by a low complexity at the phrasal, syntactic, and textual level, whereas in a text, which is for example characterized by a more complex syntax, segmentation of compounds can contribute to relieving the capacity at the morphological level (see Gutermuth 2020). Investigating this trade-off and answering the question of how these different linguistic levels interact, i.e., determining the extent to which the complexity at other linguistic levels influence the need for segmentation of compounds, certainly provides a promising avenue for future projects.

Our finding that the results on word level (Experiment 1) differ considerably from the results on sentence level (Experiment 2) was partially expected, since reading words in sentences constitutes a more natural reading environment and the results are therefore ecologically more valid than the results on word level. Following the line of reasoning in Libben et al. (2003), we thus argue that some of the discrepancies may be accounted for by "the differences between the experimental paradigms" (Libben et al. 2003: 62) in the two experiments. However, we want to point out that the experiments themselves, i.e., the analyzed eye-tracking parameters, also yielded some contradicting results. These partly contradicting gaze patterns raise some doubts as to whether data collection via eye-tracking is sufficient in answering the question of which structuring sign can more effectively assist recipients with low literacy skills in accessing compounds. In addition, when using eye-tracking it needs to be considered that eye-tracking is an online method, which allows researchers to track only what participants are focusing their attention on, but it does not allow for valid conclusions about the underlying motivation of participants' viewing behavior, i.e., it does not allow to draw conclusions about why participants keep focusing their attention on a specific word. Some of these inherent boundaries of eye-tracking might be overcome by trian- gulating data from online methods with data from offline data collection approaches, such as retrospective interviews or questionnaires. Complementing data from online methods with data from offline methods would, for example, allow researchers to explicitly consider the user perspective by asking users to evaluate in which cases, if at all, they considered the segmentation to be helpful and in which cases it hindered the reading process. Although this was beyond the scope of the study, this type of data triangulation could prove very insightful and would be a promising starting point for future research.

\section{Ethics and Conflict of Interest}

The study was approved by the ethics committee of the Deutsche Gesellschaft für Sprachwissenschaft (DGfS) (German Linguistic Society) (Note No. 2019-01-190823). The authors report no conflict of interest regarding the publication of this paper.

\section{Acknowledgements}

This research was funded by the Gutenberg Council for Young Researchers (GYR).

\section{References}

Baayen, H./Davidson, D./Bates, D. (2008): Mixed-effects modeling with crossed random effects for subjects and items. Journal of Memory and Language 59[4], 390-412.

Bates, D./Maechler, M./Bolker, B./Walker, S./Haubo B./Christensen, R./Singman, H./Dai, Bin/Scheipl, F./Grothendiek, G./Green, P./Fox, J. (2019): lme4: Linear Mixed-Effects Models using 'Eigen' and S4. Version: 1.1-21. 15.09.2021 <https://CRAN.R-project.org /package $=$ lme4>.

Bland, J./Altman, D.(1995): Multiple significance tests: the Bonferroni method. BMJ 310: 170.

Napierala, M. (2012): What Is the Bonferroni Correction? 19.09.2021 <https://docs.ufpr.br/ giolo/LivroADC /Material/S3_Bonferroni\%20Correction.pdf>.

Berg, T. (2006): The internal structure of four-noun compounds in English and German. Corpus Linguistics and Linguistic Theory 2[2]: 197-231. 
Bertram, R./Hyönä, J. (2003): The length of a complex word modifies the role of morphological structure: Evidence from eye movements when reading short and long Finnish compounds. Journal of Memory and Language 48[3]: 615-634.

Bertram, R./Hyönä, J. (2013): The Role of Hyphens at the Constituent Boundary in Compound Word Identification. Experimental Psychology 60[3]: 157-163.

Bertram, R./Kuperman, V./Baayen, H./Hyönä, J. (2011): The hyphen as a segmentation cue in triconstituent compound processing: It's getting better all the time. Scandinavian Journal of Psychology 52[6]: 530-544.

BITV 2.0 (2011): Verordnung zur Schaffung barrierefreier Informationstechnik nach dem Behindertengleichstellungsgesetz. (Barrierefreie-Informationstechnik-

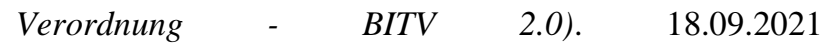
<http://www.gesetze-im-internet.de/bitv_2_0 /BJNR184300011.html>.

Blanken, G. (2000): The Production of Nominal Compounds in Aphasia. Brain and Language 74[1]: 84-102.

BMAS (2013): Leichte Sprache. Ein Ratgeber. Ed. Bundesministerium für Arbeit und Soziales. 19.09.2021 <http://www.bmas.de/SharedDocs/Downloads/DE/PDFPublikationen/a752-ratgeber-leichte-

sprache.pdf?_blob=publicationFile>.

Bredel, U./Maaß, C. (2016a): Leichte Sprache. Theoretische Grundlagen. Orientierung für die Praxis. Ed. Dudenredaktion. Berlin: Duden.

Bredel, U./Maaß, C. (2016b): Ratgeber Leichte Sprache. Die wichtigsten Regeln und Empfehlungen für die Praxis. Ed. Dudenredaktion. Berlin: Duden.

Bredel, U./Maaß, C. (2017): Wortverstehen durch Wortgliederung - Bindestrich und Mediopunkt in Leichter Sprache. „Leichte Sprache“ im Spiegel theoretischer und angewandter Forschung. Eds. B. Bock/U. Fix/D. Lange. Berlin: Frank \& Timme. 211-228.

Deilen, S. (2020): Visual segmentation of compounds in Easy Language: Eye movement studies on the effects of visual, morphological and semantic factors on the processing of German noun-noun compounds. Easy Language Research: Text and User Perspectives. Eds. S. Hansen-Schirra/C. Maaß. Berlin: Frank \& Timme. 241256.
Fahrmeir, L./Kneib, T./Lang, S. (22009): Regression. Modelle, Methoden und Anwendungen. Heidelberg: Springer.

Fox, J. (2020): Effect Displays for Linear, Generalized Linear, and Other Models. Version: 4.2-0. 15.09.2021 <https://CRAN.R-project.org/package=effects>.

Geers, A. (2003): Predictors of Reading Skill Development in Children with Early Cochlear Implantation. Ear and Hearing 24[1]: 59S-68S.

Gutermuth, S. (2020): Leichte Sprache für alle?: eine zielgruppenorientierte Rezeptionsstudie zu Leichter und Einfacher Sprache. Berlin: Frank \& Timme.

Häikiö, T./Bertram, R./Hyönä, J. (2011): The development of whole-word representations in compound word processing: Evidence from eye fixation patterns of elementary school children. Applied Psycholinguistics 32[3]: 533-551.

Hansen-Schirra, S./Maaß, C. (2020): Easy Language, Plain Language, Easy Language Plus: Perspectives on Comprehensibility and Stigmatisation. Easy Language Research: Text and User Perspectives. Eds. S. HansenSchirra/C. Maaß. Berlin: Frank \& Timme. 17-38.

Hasenäcker, J./Schroeder, S. (2019): Compound Reading in German: Effects of Constituent Frequency and WholeWord Frequency in Children and Adults. Journal of Experimental Psychology: Learning, Memory, and Cognition 45[5]: 920-933.

Hennies, J. (2009): Lesekompetenz gehörloser und schwerhöriger SchülerInnen: Ein Beitrag zur empirischen Bildungsforschung in der Hörgeschädigtenpädagogik. Dissertation. Humboldt-Universität zu Berlin. 15.09.2021 <https://edoc.hu-berlin.de/bitstream/handle/ 18452/16801/hennies.pdf?sequence $=1>$.

Holt, J. (1993): Stanford Achievement Test - 8th Edition: Reading comprehension subgroup results. American Annals of the Deaf 138[2]: 172-175.

Holt, J./Traxler, C./Allen, T. (1997). Interpreting the scores: A user's guide to the 9th Edition Stanford Achievement Test for educators of deaf and hard-ofhearing students. Washington, DC: Gallaudet University.

Inclusion Europe (2009): Informationen für alle! Europäische Regeln, wie man Informationen leicht lesbar und leicht verständlich macht. Ed. Inclusion Europe. 
20.09.2021 <https://www.lag-abt-niedersachsen.de /uploads/migrate/Download/Infofralle.pdf $>$.

Inhoff, A./Radach, R. (1998): Definition and Computation of Oculomotor Measures in the Study of Cognitive Processes. Eye guidance in reading and scene perception. Ed. G. Underwood. Oxford: Elsevier. 29-53.

Inhoff, A./Radach, R./Heller, D. (2000): Complex Compounds in German: Interword Spaces Facilitate Segmentation but Hinder Assignment of Meaning. Journal of Memory and Meaning 42[1]: 23-50.

Jaeger, T. (2009): Random effect: Should I stay or should I go? 18.09.2021 <https://hlplab.wordpress.com /2009/05/14/random-effect-structure/>.

Jaeger, T. (2011): More on random slopes and what it means if your effect is not longer significant after the inclusion of random slopes. 18.09.2021 <https://hlplab.wordpress.com/2011/06/25/more-onrandom-slopes/>.

Juhasz, B./Inhoff, A./Rayner, K. (2005): The role of interword spaces in the processing of English compound words. Language and Cognitive Processes 20[1]: 291316.

Mitchell, R./Karchmer, M. (2003): Demographic and Achievement Characteristics of Deaf and Hard-ofHearing Students. Oxford Handbook of Deaf Studies, Language, and Education. Eds. M. Marschark/P. Spencer. Oxford/New York: Oxford University Press. 21-37.

Keating, G./Jegerski, J. (2015): Experimental Designs in Sentence Processing Research. A Methodological Review and User's Guide. Studies in Second Language Acquisition 37[1]: 1-32.

Kuperman, V./Schreuder, R./Bertram, R./Baayen, H. (2009): Reading Polymorphemic Dutch Compounds: Toward a Multiple Route Model of Lexical Processing. Journal of Experimental Psychology: Human Perception and Performance 35[3]: 876-895.

Libben, G./Gibson, M./Yoon, Y./Sandra, D. (2003): Compound fracture: The role of semantic transparency and morphological headedness. Brain and Language 84[1]: 50-64.

Libben, G. (2006): Why Study Compound Processing? An overview of the issues. The Representation and Pro- cessing of Complex Words. Eds. G. Libben/G. Jarema. Oxford: Oxford University Press. 1-22.

Libben, G. (2008): How Do We Parse Compound Words? Advances in cognitive science. Ed. Narayanan Srinivasan. New Delhi: SAGE Publications. 71-86.

Lorenz, A. (2008): Die Verarbeitung von Nominalkomposita bei Aphasie. Spektrum Patholinguistik 1: 67-81.

Lorenz, A./Heide, J./Burchert, F. (2014): Compound naming in aphasia: effects of complexity, part of speech, and semantic transparency. Language, Cognition and Neuroscience 29[1]: 88-106.

Maaß, C. (2015): Leichte Sprache. Das Regelbuch. Münster: Lit-Verlag.

Maßß, C. (2020): Easy Language - Plain Language Easy Language Plus. Balancing Comprehensibility and Acceptability. Berlin: Frank \& Timme.

Marelli, M./Luzzatti, C. (2012): Frequency effects in the processing of Italian nominal compounds: Modulation of headedness and semantic transparency. Journal of Memory and Language 66[4]: 644-664.

Matuschek, H./Kliegl, R./Vasishth, S./Baayen, H./Bates, D. (2017): Balancing Type I error and power in linear mixed models. Journal of Memory and Language 94: 305-315.

O’Regan, K./Lévy-Schoen, A./Pynte, J./Brugaillère, B. (1984): Convenient fixation location within isolated words of different length and structure. Journal of Experimental Psychology: Human Perception and Performance 10[2]: 250-257.

O’Regan, K./Lévy-Schoen, A. (1987): Eye-movement strategy and tactics in word recognition and reading. Attention and Performance XII: The Psychology of Reading. Ed. M. Coltheart. Hillsdale: Lawrence Erlbaum Associates. 363-383.

Pappert, S./Bock, B. (2020): Easy-to-read German put to the test: Do adults with intellectual disability or functional illiteracy benefit from compound segmentation? Reading and Writing 33[5]: 1105-1131.

Placke, L. (2001): Das Lesen von Komposita. Blickbewegungsstudien zum Einfluss visueller, orthographischer und lexikalischer Faktoren auf die Verarbeitung komplexer deutscher und englischer Wörter. Aachen: Shaker. 
R Core Team (2018): A Language and Environment for Statistical Computing. R Foundation for Statistical Computing. 15.09.2021 <https://www.R-project.org>.

Rayner, K. (1998): Eye movements in reading and information processing: 20 years of research. Psychological Bulletin 124[3]: 372-422.

Rayner, K./Sereno, S./Raney, G. (1996): Eye Movement Control in Reading: A Comparison of Two Types of Models. Journal of Experimental Psychology: Human Perception and Performance 22[5]: 1188-1200.

Rink, I. (2020): Rechtskommunikation und Barrierefreiheit: Zur Übersetzung juristischer Informations- und Interaktionstexte in Leichte Sprache. Berlin: Frank \& Timme.

Seyboth, M. (2014): Ein Ganzes oder zwei Teile? Untersuchungen zur Verarbeitung von Komposita durch Sprachgesunde, Aphasiker und Synästhetiker. Dissertation. Universität Erfurt. 15.09.2021 <https://www.db-thueringen.de/servlets/MCRFileNodeServlet/dbt_derivate_0003 2012/Dissertation_MargretSeyboth_20150722.pdf>.

Schreuder, R./Baayen, H. (1995): Modeling Morphological Processing. Morphological Aspects of Language Processing: Cross-Linguistic Perspectives. Ed. L. Feldman. Hillsdale: Lawrence Erlbaum Associates. 131-154.

Vitu, F./O’Regan, K./Mittau, M. (1990): Optimal landing position in reading isolated words and continuous text. Perception \& Psychophysics 47[6]: 583-600.

Vitu, F./O’Regan, K./Inhoff, A./Topolski, R. (1995): Mindless reading: Eye-movement characteristics are similar in scanning letter strings and reading texts. Perception \& Psychophysics 57[3]: 352-364.

Wellmann, K. (2020): Medio-punkt oder Binde-Strich? Eine Eyetracking-Studie. Leichte Sprache - Empirische und multimodale Perspektiven. Eds. A. Gros/S. Gutermuth/K. Oster. Berlin: Frank \& Timme. 23-42.

Wolfer, S. (2016): The impact of nominalisations on the reading process: A case-study using the Freiburg Legalese Reading Corpus. Eyetracking and Applied Linguistics. Eds. S. Hansen-Schirra/S. Grucza. Berlin: Language Science Press. 163-186.

Wimmer, H./Mayringer, H. (2014/2016): SLS 2-9. Salzburger Lese-Screening für die Schulstufen 2-9. Hogrefe: Bern. 